\title{
Article \\ Molecular Identification of Keratinase DgokerA from Deinococcus gobiensis for Feather Degradation
}

\author{
Yong Meng ${ }^{1}$, Yin Tang ${ }^{2}$, Xiuhong Zhang ${ }^{1}$, Jin Wang ${ }^{2}$ and Zhengfu Zhou ${ }^{2, *}$ \\ 1 Mianyang Habio Bioengineering Co., Ltd., Mianyang 621011, China; mengyong1121@163.com (Y.M.); \\ zhangxiuhong1121@163.com (X.Z.) \\ 2 Biotechnology Research Institute, Chinese Academy of Agricultural Sciences, Beijing 100081, China; \\ tangyin15280939267@163.com (Y.T.); wangjin@caas.cn (J.W.) \\ * Correspondence: zhouzhengfu@caas.cn
}

Citation: Meng, Y.; Tang, Y.; Zhang, X.; Wang, J.; Zhou, Z. Molecular Identification of Keratinase DgokerA from Deinococcus gobiensis for Feather Degradation. Appl. Sci. 2022, 12, 464. https://doi.org/10.3390/app12010464

Academic Editor: Hoon Kim

Received: 10 December 2021

Accepted: 2 January 2022

Published: 4 January 2022

Publisher's Note: MDPI stays neutral with regard to jurisdictional claims in published maps and institutional affiliations.

Copyright: (C) 2022 by the authors. Licensee MDPI, Basel, Switzerland. This article is an open access article distributed under the terms and conditions of the Creative Commons Attribution (CC BY) license (https:// creativecommons.org/licenses/by/ $4.0 /)$.

\begin{abstract}
Keratin is a tough fibrous structural protein that is difficult to digest with pepsin and trypsin because of the presence of a large number of disulfide bonds. Keratin is widely found in agricultural waste. In recent years, especially, the development of the poultry industry has resulted in a large accumulation of feather keratin resources, which seriously pollute the environment. Keratinase can specifically attack disulfide bridges in keratin, converting them from complex to simplified forms. The keratinase thermal stability has drawn attention to various biotechnological industries. It is significant to identify keratinases and improve their thermostability from microorganism in extreme environments. In this study, the keratinases DgoKerA was identified in Deinococcus gobiensis I-0 from the Gobi desert. The amino acid sequence analysis revealed that DgoKerA was $58.68 \%$ identical to the keratinase MtaKerA from M. thermophila WR-220 and 40.94\% identical to the classical BliKerA sequence from $B$. licheniformis PWD-1. In vitro enzyme activity analysis showed that DgoKerA exhibited an optimum temperature of $60^{\circ} \mathrm{C}$, an optimum $\mathrm{pH}$ of 7 and a specific enzyme activity of $51147 \mathrm{U} / \mathrm{mg}$. DgoKerA can degrade intact feathers at $60^{\circ} \mathrm{C}$ and has good potential for industrial applications. The molecular modification of DgoKerA was also carried out using site-directed mutagenesis, in which the mutant A350S enzyme activity was increased by nearly $30 \%$, and the results provide a theoretical basis for the development and optimization of keratinase applications.
\end{abstract}

Keywords: Deinococcus gobiensis; keratinase; thermostability; feather degradation; site-directed mutation

\section{Introduction}

Keratin is rich in nutrients and contains up to $70 \%$ of various amino acids, such as cysteine, lysine, proline and serine, which makes it a potentially valuable protein [1]. Keratin is difficult to degrade and utilize due to the strong properties conferred by the huge cross-linking of disulfide and hydrogen bonds, and the recycling of keratin of animal origin, which is produced by the poultry industry at over 1 billion tons per year, has become a challenge for the industry [2,3]. Keratinase acts specifically on the disulfide bonds of keratin and, thus, hydrolyses keratin [4]. Keratinases are widely found in microorganisms and the use of keratinases to recover keratin-related resources is an environmentally friendly, low energy consuming and sustainable approach [5].

Keratin hydrolases or keratinases (EC 3.4.21/24/99.11) are classified as serine proteases, aspartate proteases, metalloproteases and serine-metal complex proteases, depending on the inhibition of the amino acid group in the active site of the protease [4]. Among them, the serine keratinase group is the most numerous and widely used, represented by Bacillus keratinases. Bacillus licheniformis PWD-1is able to grow and ferment in feather digesters in both anaerobic and aerobic environments [6]. BliKerA, derived from B. licheniformis PWD-1 keratinase, is the most well-studied keratinase. BlikerA has been successfully expressed in different hosts, such as Bacillus subtilis, Bacillus megaterium, and 
Pichiia pastoris, it has been developed into the commercial enzyme "Versazyme" and is widely used as a nutrient preparation. The keratinase sequence contains a signal peptide, an $\mathrm{N}$-terminal propeptide and a mature region, and some keratinases contain $\mathrm{C}$-terminal extensions [7]. B. licheniformis PWD-1 keratinase BliKerA contains only the signal peptide, the N-terminal prepeptide and the mature region, whereas Thermococcus kodakaraensis keratinase ProN-Tk-SP contains these three structures in addition to the C-terminal extension structure $[6,8]$. The signal peptide directs the secretion of the keratinase to its extracellular function, while the N-terminal propeptide usually acts as an intramolecular chaperone to facilitate the correct folding of the keratinase structure [9]. The major structures of the keratinase mature region are the catalytic active center and the substrate recognition site. The activity center contains the Asp-His-Ser catalytic triad. The gap adjacent to the three catalytic residues becomes the S1 pocket, and the spatial structure and hydrophobicity of the S1 pocket affects the catalytic activity of keratinases $[10,11]$. In addition, the enzymes generally contain calcium-binding sites, which contribute to the thermal stability of keratinases [3,12].

Extremophiles are considered a valuable source for the production of novel enzymes due to their unique metabolism and ability to withstand extreme environments, such as high temperature, high pressure, high $\mathrm{pH}$ and high radiation [13-15]. Many studies have been conducted to identify keratinases with excellent properties from extremophiles, such as Nocardiopsis [16], Salicola Marasensis [17] and Meiothermus taiwanensis WR-220 [7]. M. taiwanensis WR-220 was a thermophilic microorganism with a growth temperature of $55-65{ }^{\circ} \mathrm{C}$. The keratinase from this bacterium also exhibited thermal stability, maintaining intact enzymatic activity after $1 \mathrm{~h}$ of treatment at $75^{\circ} \mathrm{C}$ [7]. It was shown that keratinase genes are present in extreme microorganisms Deinococcus [14], such as D. geothermalis [18] and Deinococcus sp. D7000 [19], and that the feather-degrading bacteria D. ficus [20] and $D$. radiodurans $\mathrm{R} 1$ [14] are able at $30^{\circ} \mathrm{C}$ to degrade intact feathers.

In this study, the keratinase DgoKerA was identified from the Gobi-derived D. gobiensis I-0 by comparative genomic analysis. Structurally, DgoKerA possessed a signal peptide, an $\mathrm{N}$-terminal propeptide and a mature region, as did the classical keratinase BliKerA. The enzymatic properties and feather degradation ability of the recombinant keratinase were determined by expression and purification with E. coli. DgoKerA was further modified by site-directed mutation to lay the foundation for its industrial application.

\section{Materials and Methods}

\subsection{Bacterial Strains, Plasmids, and Materials}

The strain Deinococcus gobiensis I-0 was cultured at $30^{\circ} \mathrm{C}$ for $12 \mathrm{~h}$ in TGY medium [21]. The E. coli strain BL21 (DE3) was cultured in Luria-Bertani (LB) medium at $37^{\circ} \mathrm{C}$. Kanamycin $(50 \mathrm{~g} / \mathrm{mL})$ was added for plasmid selection. The vector pET28a was used for the expression of keratinase genes in E. coli. All of the enzymes for DNA manipulations were purchased from Vazyme (Nanjing, Jiangsu, China). All chemicals used in this study were of reagent grade.

\subsection{Construction of Keratinase Expression Plasmid}

The DgoKerA gene without the signal sequence was obtained by amplification. Primer sequences designed using the genome of D. gobiensis as a template are shown in Table 1. The amplified fragments were cloned into pET28a expression vector (Novagen) containing the C-terminal His-tag using ClonExpress Ultra One Step Cloning Kit (Vazyme). The correctly recombinant plasmids were transformed into E. coli BL21 (DE3) competent cells for gene expression. The mutation recombination vector is constructed using the principle of homologous recombination. The mutant gene fragment is divided into two segments from the targeted mutation site, and the two segments are amplified by PCR separately. The different DNA fragments and vector can be complementary paired by homologous arms of the same sequence to assemble the expression vectors using homologous recombinase. 
Table 1. Primers used in this study.

\begin{tabular}{cr}
\hline Name of Primers & Sequences $\mathbf{~}^{\prime}{ }^{\prime}$ to $\mathbf{3}^{\prime} \mathbf{)}$ \\
\hline DgokerA-F & aaggagatataccatgggcATGTGCGGAACCTCGACTACCC \\
DgokerA-R & gtggtggtggtggtgctcgagGAAGTTCAGGGTGTACAGCAGCT \\
A350S-F1 & aggagatataccatgggcATGTGCGGAACCTCGACTACCC \\
A350S-R1 & gtgccgctgatggtgttggtGCTGGTCGTGCTGCCGAT \\
A350S-F2 & gatcggcagcacgaccagcACCAACACCATCAGCGGCA \\
A350S-R2 & gtggtggtggtggtgctcgagGAAGTTCAGGGTGTACAGCAGCT \\
V299F-F1 & aggagatataccatgggcATGTGCGGAACCTCGACTACCC \\
V299F-R1 & ctggcacgcgccgggctGAAGTTGCAGGCGTCCTGG \\
V299F-F2 & aggacgcctgcaacttcAGCCCGGCGCGTGCCAGC \\
V299F-R2 & gtggtggtggtggtgctcgagGAAGTTCAGGGTGTACAGCAGCT \\
\hline
\end{tabular}

The lower case letters of primer sequences represent homologous arm sequences; red marked bases represent mutant bases.

\subsection{Expression and Purification of Keratinases}

The recombinant E. coli was cultured overnight at $37^{\circ} \mathrm{C}$ in LB medium containing $50 \mu \mathrm{g} / \mathrm{mL}$ of Kanamycin. The cultures were transferred into $150 \mathrm{~mL}$ of fresh medium and incubated at $37^{\circ} \mathrm{C}$ until $\mathrm{OD}_{600}=0.6$, and then $0.5 \mathrm{mM}$ isopropyl- $\beta$-D-thiogalactoside (IPTG) was added for $16 \mathrm{~h}$ at $16^{\circ} \mathrm{C}$. The cells were harvested by centrifugation at $4000 \times g$ for $15 \mathrm{~min}$ and resuspended with Tris- $\mathrm{HCl}(\mathrm{pH} 8.0)$, and disrupted on ice by sonication for $10 \mathrm{~min}$. The debris was removed by centrifugation at $12,000 \times \mathrm{g}$ for $30 \mathrm{~min}$ at $4{ }^{\circ} \mathrm{C}$. The recombinant keratinases were purified by Ni-affinity chromatography (Qiagen) and eluted with an increasing imidazole gradient. The molecular weight of the purified DgoKerA and mutants were analyzed by SDS-PAGE.

\subsection{Keratinolytic Activity Assay}

Keratinolytic activity was assayed with soluble keratin according to Tang' s protocol [18]. The reaction mixture, which contained $20 \mu \mathrm{L}$ enzyme, $80 \mu \mathrm{L}$ buffer and $100 \mu \mathrm{L}$ soluble keratin $(1 \%, w / v)$, was incubated at $50{ }^{\circ} \mathrm{C}$ for 20 min. A blank control was conducted by adding $200 \mu \mathrm{L}$ of $20 \%(w / v)$ trichloroacetic acid (TCA) instead of soluble keratin (Tokyo Chemical Industry Co. Ltd., Tokyo, Japan). The reaction was terminated by the addition of $200 \mu \mathrm{L} \mathrm{TCA}$, and centrifuged at $12,000 \times g$ for $10 \mathrm{~min}$. An amount of $100 \mu \mathrm{L}$ soluble keratin was added to the control mixture. Then, $200 \mu \mathrm{L}$ supernatant was pipetted into another tube with $1 \mathrm{~mL} 0.4 \mathrm{~mol} / \mathrm{L} \mathrm{Na}_{2} \mathrm{CO}_{3}$ and $200 \mu \mathrm{L}$ Folin-Phenol regent. Finally, the mixtures were incubated at $40^{\circ} \mathrm{C}$ for $20 \mathrm{~min}$ to develop the color. Proteolytic products in the mixture were measured spectrophotometrically at $\mathrm{OD}_{680}$. One unit $(\mathrm{U})$ of keratinase activity was defined as an increase of 0.01 absorbance unit at $\mathrm{OD}_{680}$ under the conditions above mentioned.

To determine the activity of feather powder degradation, Yang's method was carried out with slight modifications [22]. The reaction system, including $4 \mathrm{~mL}$ buffer, $0.01 \mathrm{~g}$ feather powder and $200 \mu \mathrm{L}$ enzyme with $5 \mathrm{mmol} / \mathrm{L}$ dithiothreitol (DTT), was incubated at $60{ }^{\circ} \mathrm{C}$ for $60 \mathrm{~min}$ and subsequently terminated by adding $2.0 \mathrm{~mL}$ of $20 \%$ TCA. After centrifugation at $12,000 \times g$ for $5 \mathrm{~min}$, the supernatant was gathered for measuring the absorbance at $\mathrm{OD}_{280}$. TCA was added before the enzymatic reaction as the control. One unit (U) of feather powder activity was defined as an increase of 0.01 absorbance unit $\mathrm{OD}_{280}$. Protein concentration was determined using the Bradford method with bovine serum albumin as a standard. All assays were performed in triple.

\subsection{Effects of $\mathrm{pH}$, Temperature, and Reagents on Enzyme Activity and Stability}

The optimal reaction temperature of DgoKerA was investigated in the temperature range $30-80{ }^{\circ} \mathrm{C}$, and the optimal $\mathrm{pH}$ experiment was carried out over a $\mathrm{pH}$ range of 3-11 (pH 3.0-6.0, 50 mmol/L citrate buffer; $\mathrm{pH}$ 7-9, 50 mmol/L Tris-HCl; $\mathrm{pH}$ 10-11, $50 \mathrm{mmol} / \mathrm{LGly}-\mathrm{NaOH}$ ), using $1 \%$ soluble keratin. In thermostability assays, enzymes were pre-incubated at $60^{\circ} \mathrm{C}$ for $60 \mathrm{~min}$, and residual enzyme activities were determined. The 
$\mathrm{pH}$ stability was measured by pre-incubating DgoKerA in different $\mathrm{pH}$ solutions at $4{ }^{\circ} \mathrm{C}$ for $1 \mathrm{~h}$, and then the residual activities were detected.

The effect of metal ions on the enzyme activity was analyzed by assaying the relative activity with addition of $5.0 \mathrm{mmol} / \mathrm{L}$ of metal ions $\left(\mathrm{K}^{+}, \mathrm{Li}^{+}, \mathrm{Mn}^{2+}, \mathrm{Cu}^{2+}, \mathrm{Fe}^{2+}, \mathrm{Ca}^{2+}, \mathrm{Co}^{2+}\right.$, $\left.\mathrm{Mg}^{2+}, \mathrm{Ni}^{2+}, \mathrm{Cd}^{2+}, \mathrm{Cr}^{3+}\right)$. Similarly, the effects of some surfactants and organic solvent on enzyme activity were also analyzed. 1\% Dimethyl sulfoxide (DMSO), $1 \% \beta$-mercaptoethanol, $1 \%$ Tween-20, $1 \%$ Tween- 80 and $1 \%$ SDS Sodium dodecyl sulfate (SDS) $(v / v)$ were added for the relative activity evaluation. The results were analyzed with SPSS Statistics 25 software. The differences in means between groups were compared for statistical significance at $p<0.05$.

\subsection{Chicken Feather Degradation}

The degradation of chicken feathers was carried out according to Ren's method [23], with some modifications. Chicken feathers were previously washed and cleaned with detergent and tap water, followed by soaking in $70 \%$ ethanol for $1 \mathrm{~h}$ and drying. After this, the feathers were autoclaved at $121^{\circ} \mathrm{C}$ for $30 \mathrm{~min}$ to remove microorganism on degradation of feathers. The intact chicken feathers were incubated with $0.06 \mathrm{mg}$ enzyme at $60{ }^{\circ} \mathrm{C}$ with $5 \mathrm{mmol} / \mathrm{L}$ DTT. The degradation process of feathers was recorded regularly.

\section{Results}

\subsection{Identification of Keratinase DgoKerA}

The gene dgoKerA (1239 bp, DGo_CA0576), derived from D. gobiensis I-0, encodes a protein comprising 412 amino acids with a calculated mass of $41.17 \mathrm{kDa}$. Phylogenetic analysis of DgoKerA and other homologous keratinases was constructed based on the amino acid sequence. As shown in Figure 1, The DgoKerA showed significant relatedness to peptidase from genus Deinococcus; they were localized at the same branch in the phylogenetic tree, indicating a common evolutionary origin. The protein sequence of DgoKerA displayed similarity to MtaKerA from M. taiwanensis WR-220 (AWR86689.1) with 58.68\%. DgoKerA had a certain homology with the keratinases from the Thermus, such as aqualysin I (WP_053768483.1) from Thermus aquaticus and peptidase (WP_172955617.1) from Thermus scotoductus with $54.84 \%$ and $57.44 \%$ identity, respectively. The amino acid sequence of DgoKerA, compared with those of keratinases, were BliKerA from Bacillus licheniformis PWD-1, and MtaKerA from M. taiwanensis WR-220, as seen in Figure 2. It was proposed that DgoKerA consists of three parts, including signal peptide (pre), N-terminal pro-peptide (N-pro), and the mature domain with a three highly conserved catalytic triad residues which is formed by Asp171, His203 and Ser358 (black triangle). The results of phylogenetic tree and sequence identity analysis indicated that DgoKerA is a keratinase.

\subsection{Expression and Activity of DgoKerA}

In order to obtain purified DgoKerA, a recombinant plasmid carrying keratinase gene without signal peptide was constructed for the E. coli expression system. DgoKerA was expressed in E. coli BL21 (DE3) strains, purified on a Ni-column, and dialyzed against $40 \mathrm{mM}$ Tris $-\mathrm{HCl}$ ( $\mathrm{pH}$ 8.0). The protein bands detected in the SDS-PAGE after purification migrated at approximately $28 \mathrm{kDa}$, which corresponds to the molecular mass of the mature keratinases (Figure 3). The MALDI-TOF MS results showed that the purified protein did not contain the N-propeptide sequence. This indicates that the DgoKer N-propeptide is cleaved normally in E. coli and that the purified product contains only the mature region keratinase. In this study, $1 \%$ soluble keratin and feather powder were used as substrates to measure the keratinase activity. The keratinase DgoKerA specific enzyme activity was $51,147 \pm 442.80 \mathrm{U} / \mathrm{mg}$ with $1 \%$ soluble keratin as the substrate, as shown in Table 2. In addition, the DgoKerA activity was $3277.88 \pm 172.62 \mathrm{U} / \mathrm{mg}$ with feather powder as the substrate. 


\subsection{Effects of Temperature, $p H$, Metal Ions, and Chemical Agents on DgoKerA Activity}

A $1 \%$ soluble keratin was used to measure the enzyme activity. As shown in Figure 4, the optimal temperature of DgoKerA was $60^{\circ} \mathrm{C}$ in $50 \mathrm{mmol} / \mathrm{L}$ Tris- $\mathrm{HCl}$ buffer ( $\left.\mathrm{pH} 7.0\right)$. The thermostability of DgoKerA was characterized. The residual activity of DgoKerA was approximately $78 \%$ after $30 \mathrm{~min}$ incubation at $60{ }^{\circ} \mathrm{C}$ and approximately $40 \%$ after 60 min incubation. DgoKerA showed maximum activity at around $\mathrm{pH} 6-8$. DgoKerA was sensitive in the range of $\mathrm{pH} 3-4$, and was stable in range of 5-9. The residual activity of DgoKerA was approximately $88 \%$ when incubated at $\mathrm{pH} 6$ for $60 \mathrm{~min}$. Based on Figure 4, the keratinase BliKerA activity was lost more rapidly under $60^{\circ} \mathrm{C}$ treatment, with less than $20 \%$ residual activity after $60 \mathrm{~min}$ incubation. The date appears to suggest that DgoKerA has the potential of industry application, due to the outstanding thermostability.

The effects of various metal irons on keratinolytic activity were shown in Figure 5A. $\mathrm{K}^{+}, \mathrm{Li}^{+}, \mathrm{Mg}^{2+}$ and $\mathrm{Co}^{2+}$ increased the enzyme activity of DgoKerA. Other tested metal ions slightly detected activity of DgoKerA, except $\mathrm{Cr}^{3+}$, which markedly decreased the activity of DgoKerA. All of the surfactants and organic solvent employed negatively impacted on the stability of DgoKerA, with more drastic effect obtained in the presence of $\beta$-mercaptoethanol. The good tolerance of DgoKerA to organic solvent DMSO and surfactant (Tween-80 and Tween-20) was discovered (Figure 5B).

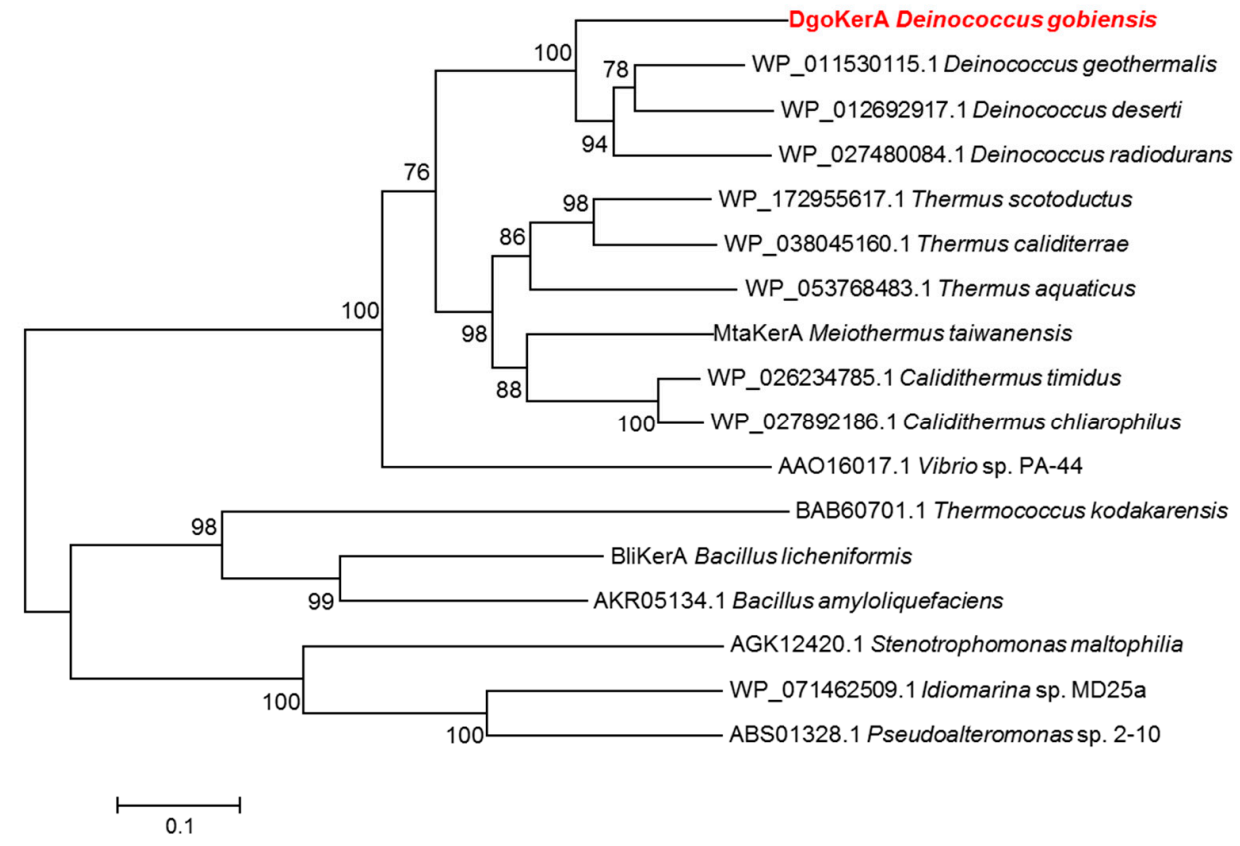

Figure 1. Phylogenetic analysis of amino acid sequence of keratinases. The name and GenBank accession No. for each protease are in front of the Latin name of each strain. The phylogenetic tree was constructed by MEGA 6.0 with neighbor-joining (NJ) method. The percentages of replicate trees in which the associated taxa clustered together in the bootstrap test (1000 replicates) are shown next to the branches. 


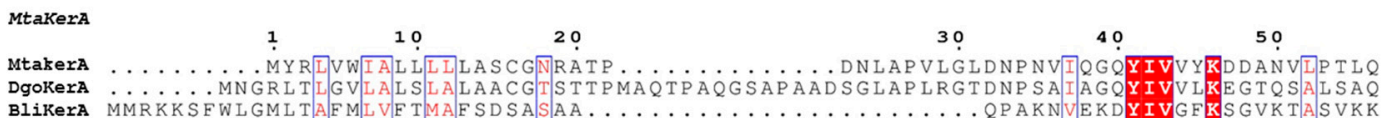

Bliker MMRKKSFWLGMLT GVILILALAACG STTPMAQTPAQGSAPAADSGLAPLRGTDNP SAIAGQYIVVLKEGTQSALSAQ
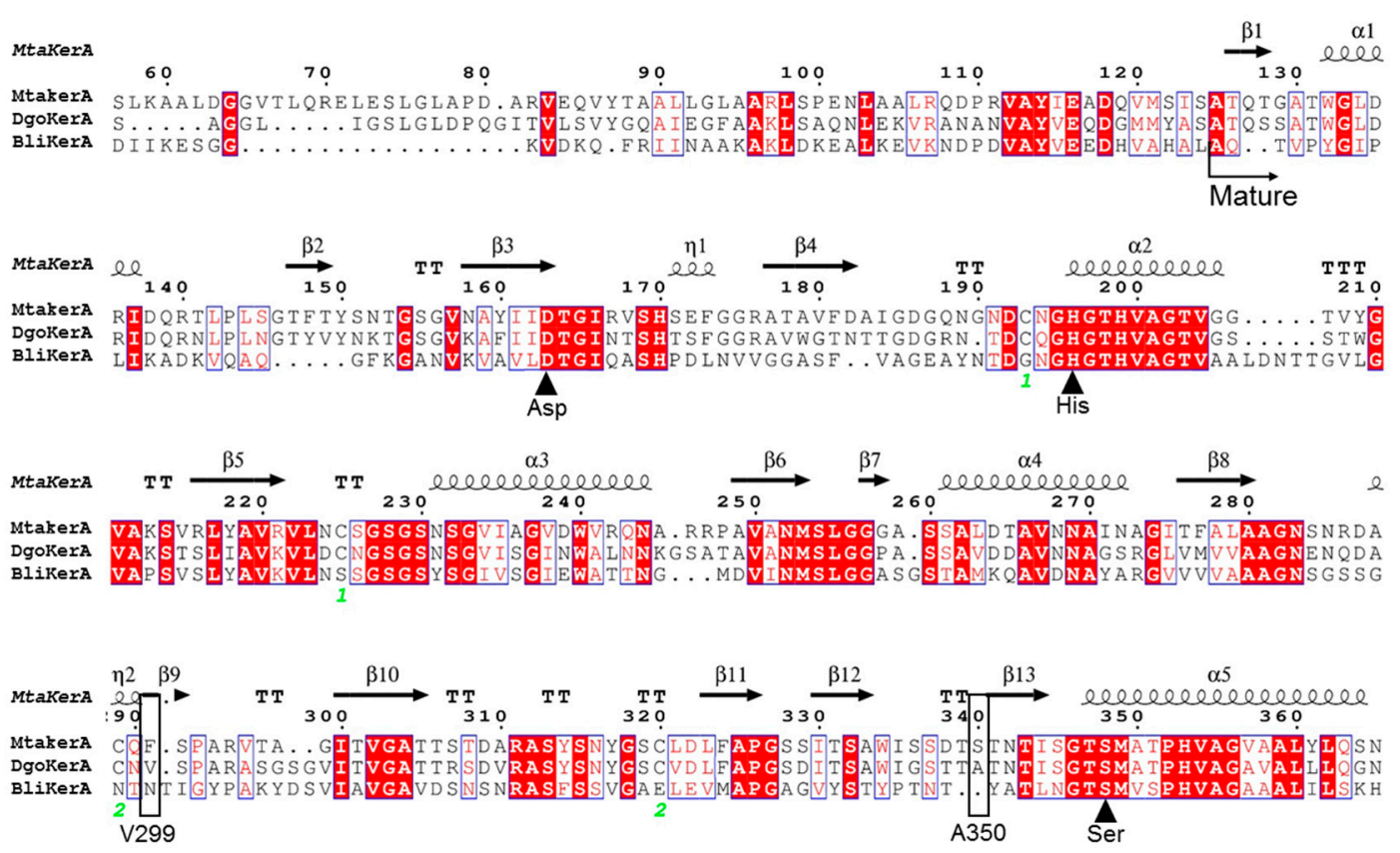

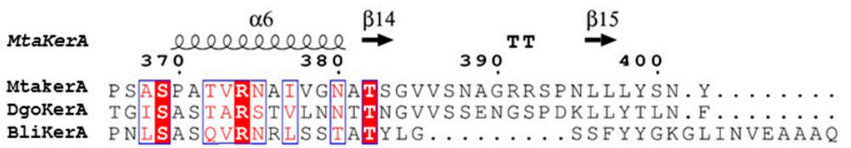

Figure 2. Alignment of the amino acid sequences of DgoKerA. The keratinases used were MtaKerA from M. taiwanensis WR-220 and BliKerA from B. licheniformis PWD-1. Sequence alignment showing structural elements of DgoKerA were generated with ESPript3.0 (https: / / espript.ibcp.fr/ESPript/ cgi-bin/ESPript.cgi (accessed on 7 November 2021)). The identical residues of all aligned proteins are shaded red. $\alpha, \beta, \eta$, TT above the sequences represent $\alpha$ helix, $\beta$ sheet, 310 -helices and $\beta$-turns, respectively. Positions of the starting residues of the mature protease (mature) was marked by black arrows at the bottom of the sequences.

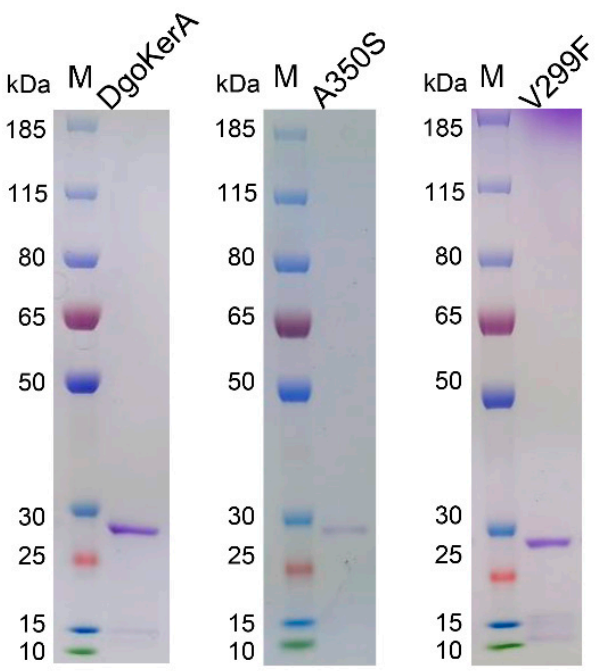

Figure 3. SDS-PAGE analysis of purified recombinant keratinase DgoKerA and its mutants. M means the protein standard marker line. 
Table 2. The enzymatic activities of several keratinases on $1 \%$ soluble keratin and feather powder.

\begin{tabular}{ccccc}
\hline \multicolumn{5}{c}{ The Enzymatic Activity (U/mg) } \\
\hline & BliKerA & DgoKerA & DgoKerA A350S & DgoKerA V299F \\
\hline $1 \%$ Soluble Keratin & $45,730.65 \pm 244.30$ & $51,147.65 \pm 442.80$ & $66,872.84 \pm 137.41$ & $49,367.23 \pm 346.51$ \\
\hline Feather Powder & $1771.63 \pm 25.29$ & $3277.88 \pm 172.62$ & $3312.14 \pm 201.10$ & $1914.17 \pm 156.42$ \\
\hline
\end{tabular}

A

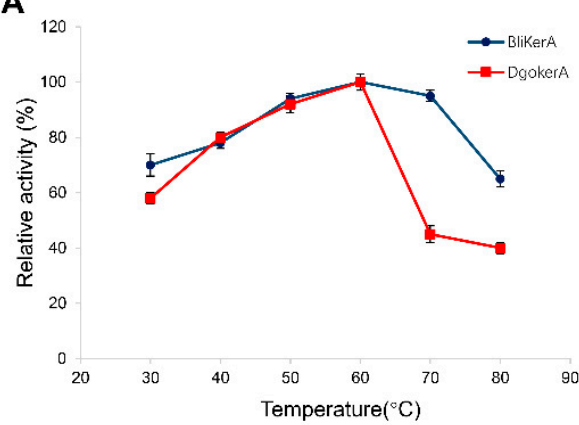

C $_{120}$

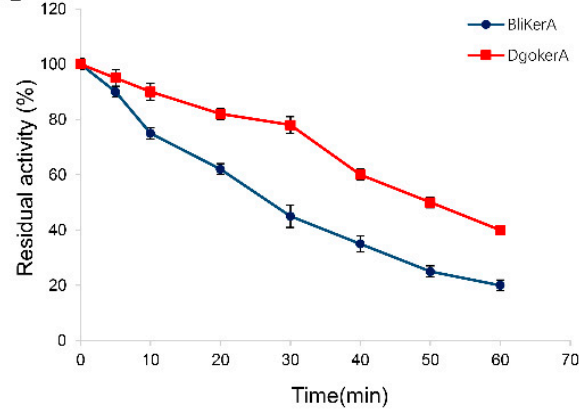

B

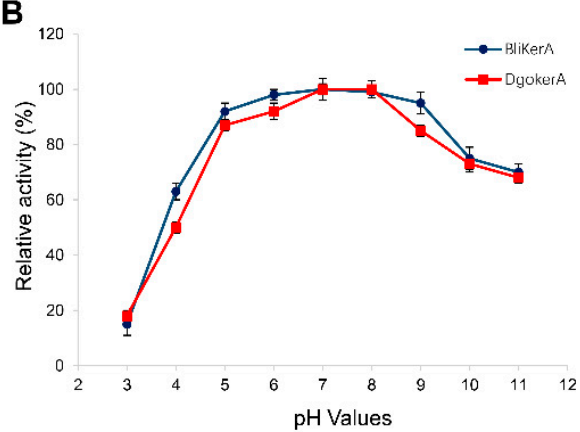

$D_{100}$

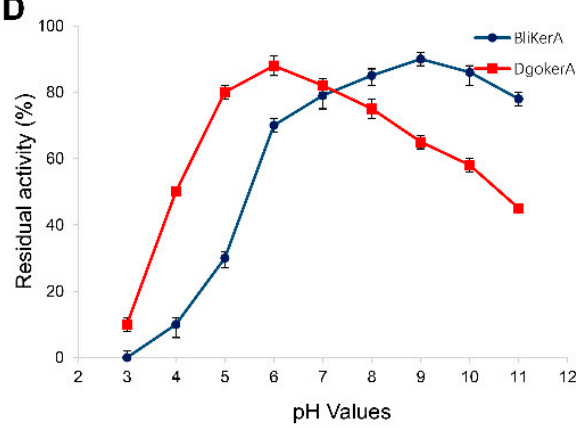

Figure 4. Effects of temperature and $\mathrm{pH}$ on keratinase activity. (A) effects of temperature for DgoKerA and BliKerA; (B) effects of $\mathrm{pH}$ for DgoKerA and BliKerA; (C) the thermostability of keratinase; (D) the $\mathrm{pH}$ stability of keratinase.

A

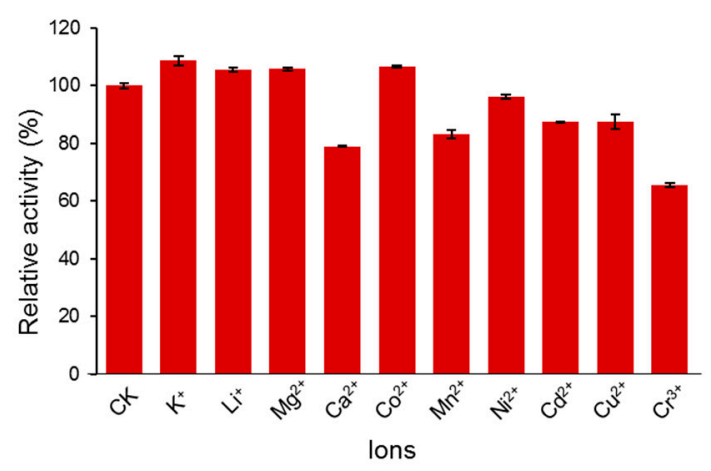

B

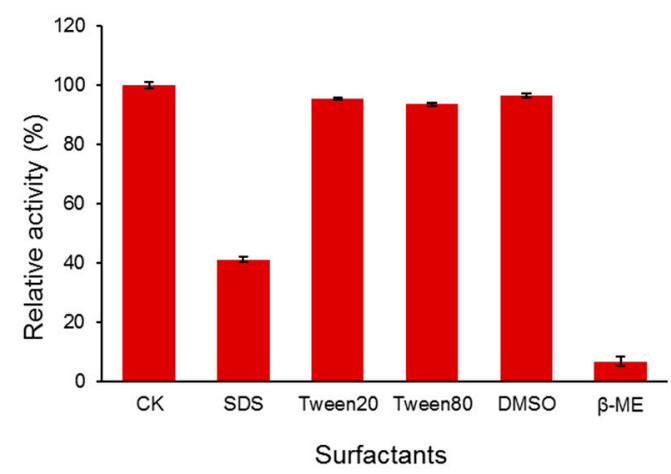

Figure 5. Effects of inorganic ions and chemicals on keratinolytic activity of DgoKerA. (A) Effects of various metal ions on keratinase activity; (B) effects of chemicals on keratinase activity. Each value was repeated in triple independent measurements.

\subsection{Chicken Feather Degradation of DgoKerA In Vitro}

In the study, the degradation of intact feathers by the keratinase DgoKerA was tested at $60{ }^{\circ} \mathrm{C}$. A Tris- $\mathrm{HCl}$ buffer without keratinase was used as a control. Figure 6 shows that the feather branches were visibly shed with $60 \mathrm{~min}$ incubation, and the barbules on the feathers were largely degraded by DgoKerA with 90 min incubation and the reaction 
system solution became turbid. The feathers are largely degraded by 120 min incubation, with only the feather rachis left. In summary, the DgoKerA has the ability to degrade intact feathers.
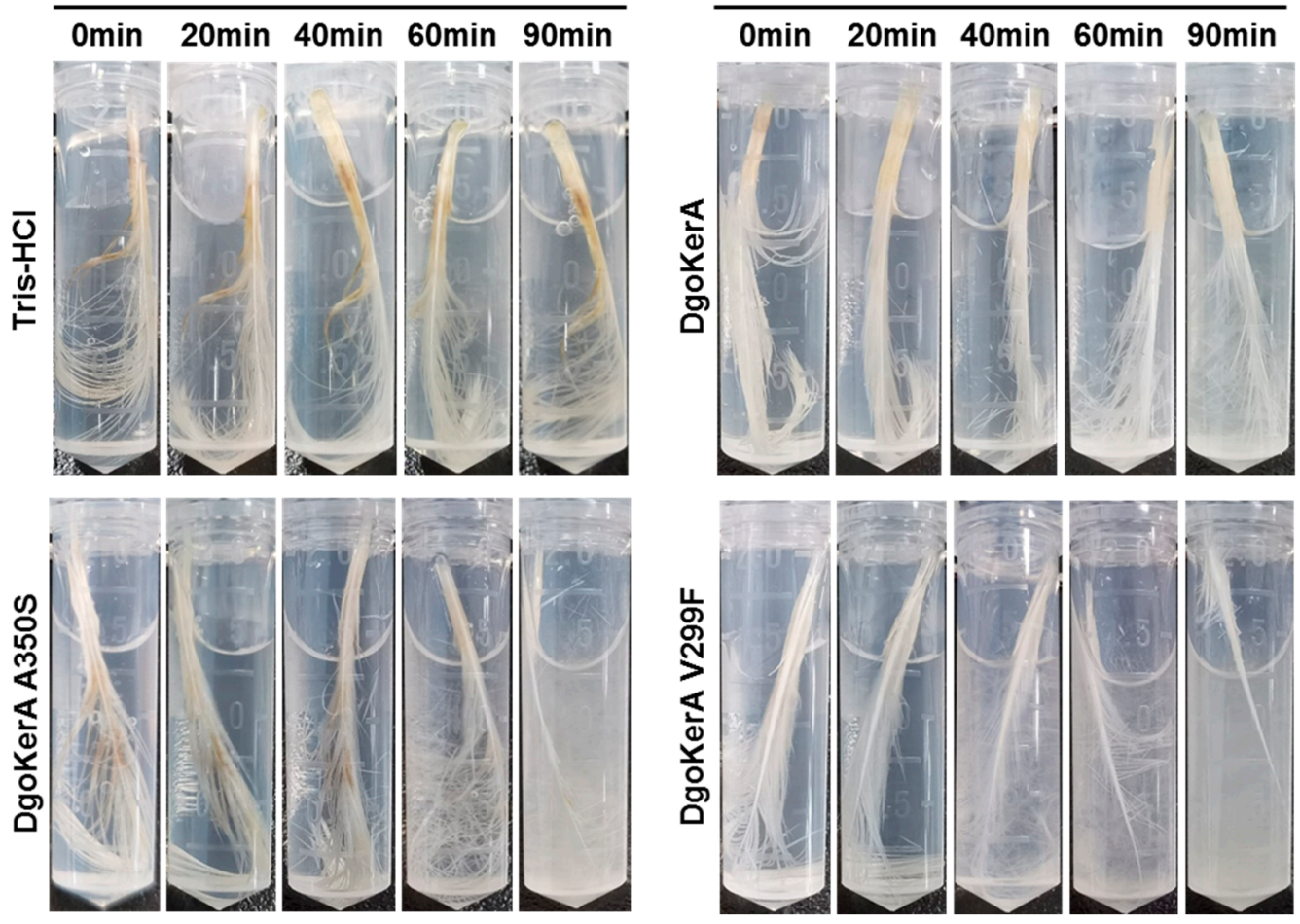

Figure 6. Comparison of intact feather degradation capacities of DgoKerA and mutants. Tris- $\mathrm{HCl}$ is a control without keratinase. The reaction systems were incubated at $60{ }^{\circ} \mathrm{C}$ with shaking.

\subsection{Enzymatic Activity and Feather Degradation Capacity of Mutants}

In this study, we obtained mutation sites and substituted amino acid residues through sequence alignment and homologous protein mutation experiments. We selected the amino acid residue V299 near the S1 substrate binding pocket and the residue A350 in the loop region linking the catalytic triad for mutagenesis. The most frequently encountered amino acid residues in the corresponding sites 299 and 350 of the homologous protease are phenylalanine and serine, respectively. Thus, A350S and V299F were selected for site-directed mutagenesis of DgoKerA.

The enzymatic activities of DgoKerA mutants of were measured with $1 \%$ soluble keratin and feather powder as substrates. As shown in Table 2, the enzymatic activity of the mutant A350S was higher than that of DgoKerA $(66,872.84 \pm 137.41 \mathrm{U} / \mathrm{mg}$ vs. $51,147.65 \pm 442.80 \mathrm{U} / \mathrm{mg}$ ) using $1 \%$ soluble keratin as the substrate, with an increase of about $30 \%$. In addition, mutant V299F also exhibited high keratinolytic activity on soluble keratin and feather powder.

The degradation of whole-feathers by each mutant at $60{ }^{\circ} \mathrm{C}$ is shown in Figure 6 . The mutants A350S and V299F all exhibited better feather degradation compared with that of DgokerA. The feather branches were clearly shed by the treatment of V299F for $40 \mathrm{~min}$ and the solution changed from clarified to turbid. Subsequently, more feathers were decomposed to small debris in the reaction system. After 90 min of incubation, the whole-feathers were degraded by V299F to just rachis.

\section{Discussion}

The extremophiles are an important source for the industrial enzymes with excellent properties [24]. In this study, the keratinase DgoKerA was identified by comparative genomic analysis from $D$. gobiensis I-0 from the Gobi desert. The phylogenetic tree showed that DgoKerA is consistent with the evolutionary origin of Deinococcus keratinase and is closely 
related to the thermotolerant bacteria Calidithermus and Thermus keratinase, suggesting that DgoKerA may have better thermal stability. Sequence alignment shows that DgoKerA, like the classical keratinase BliKerA, contains a signal peptide, an N-terminal propeptide and a mature region. The $\mathrm{N}$-propeptide of keratinase functions as an intramolecular chaperone to facilitate the folding of keratinase, and is cleaved off by mature keratinase in order to function $[9,25,26]$. Using the E. coli expression system, DgoKerA was successfully expressed and purified. the molecular weight of the purified DgoKerA was consistent with the predicted size of the protein after cleavage of the N-propeptide, indicating that DgoKerA was correctly folded and present as a mature enzyme in E. coli, as with other exogenously expressed keratinases [27,28].

The thermal stability of keratinase is important for feather degradation [29]. DgoKerA showed optimal enzymatic activity at $60{ }^{\circ} \mathrm{C}$, and the higher temperature adaptation of this enzyme may be related to the high temperature environment of the Gobi where it originated from. It was shown that the half-life (t1/2) of DgoKerA at $60{ }^{\circ} \mathrm{C}$ is 1.76 folds higher than that of BliKerA (46.52 min vs. $26.46 \mathrm{~min}$ ). The sequence analysis showed that the DgokerA mature region contains four cysteine residues. The homology modeling results using MtaKerA (PDB ID: 5wsl.1) as a template showed that two intramolecular disulfide bonds, Cys200-Cys232 and Cys297-Cys330, were formed in the structure of DgoKerA, which enhanced the keratinase thermal stability. An amount of $5 \mathrm{mmol} / \mathrm{L} \mathrm{K}^{+}$ and $\mathrm{Li}^{+}$increased the enzymatic activity of DgoKerA, while $5 \mathrm{mmol} / \mathrm{L} \mathrm{Mg}^{2+}$ and $\mathrm{Co}^{2+}$ also promoted the degradation of soluble keratin by DgoKerA. In general, certain metal ions can stabilize the conformation of the enzyme-substrate complex, thereby enhancing keratinase activity [30-33].

At present, most reported keratinases are alkaline and neutral proteases with an optimum $\mathrm{pH}$ value of 7.5-9.0. DgoKerA showed stable activity at pH 5-9. The residual activity of DgoKerA was $88 \%$ with incubated at pH 6 for 60 min and was $65 \%$ when incubated at $\mathrm{pH} 9$ for $60 \mathrm{~min}$. Extracellular enzymes encoded by microbes need to be evolved to survive in the environment [7]. Compared with BliKerA isolated from a poultry waste digester, DgoKerA, which was cloned from a high-temperature and arid environment, was more sensitive to $\mathrm{pH}$ changes.

The results of the study also showed that DgoKerA had a good tolerance to chemical reagents and the residual enzyme activity of DgoKerA remained above $90 \%$ after Tween 80 , Tween 20 and DMSO treatment for $1 \mathrm{~h}$. Non-specific binding between the ionic surfactant SDS and the protein inhibits the activity of DgoKerA, probably due to complex formation and protein defolding [34]. Reducing agents protect the free sulfhydryl groups on proteins from oxidation, thus avoiding aggregation or denaturation of proteins [35]. However, the reductant $\beta$-mercaptoethanol significantly inhibited the activity of DgoKerA on soluble keratin, with only $6.73 \%$ residual enzyme activity.

To test the ability of the keratinase DgoKerA to degrade feathers, a final concentration of $5 \mathrm{mmol} / \mathrm{L}$ DTT was added to the enzyme-feather mixture $(0.03 \mathrm{mg}$ purified enzyme, $1.5 \mathrm{~mL}$ ) in the present work. Keratin substances, such as feathers, are exceptionally strong due to their richness in disulfide bonds, hydrogen bonds and hydrophobic interactions, and most purified keratinases are unable to complete degradation. In this study, purified DgoKerA did not degrade intact feathers quickly enough without DTT, and it is possible that the enzyme does not have a strong reductive capacity. It has been shown that the reductants DTT [36], $\mathrm{Na}_{2} \mathrm{SO}_{3}$ [37] and L-cysteine [38] can all work synergistically with keratinase to accelerate the degradation of feather keratin. In this paper, we found that DgoKerA can degrade intact feathers in the presence of $5 \mathrm{mmol} / \mathrm{L}$ DTT and has good potential for industrial applications.

We also modified the keratinase DgoKerA using a combination of homology comparisons and site-directed mutagenesis. Mutant A350S and V299F have degrading activity against soluble keratin and the optimum temperature of reaction is consistent with that of WT DgoKerA. In addition, both mutant keratinases showed better degradation of intact feathers at $60{ }^{\circ} \mathrm{C}$. The amino acid residue Val 299 is lying at the entrance of the S1 pocket 
where the substrate binds. Zhen Fang et al. mutated amino acid residues associated with the keratinase FDD substrate binding site and found that Y215F increased the enzyme activity by altering the keratinase substrate specificity [29]. The mutation of the valine residue at position 299 to phenylalanine in this study alters the spatial structure of the pocket channel, and its increased feather degradation ability is likely caused by altering the substrate binding specificity.

The helix $\alpha 5$ is the region where the catalytic center Ser357 is located and is closely related to the stability of the substrate channel and the active pocket [2,39]. Position Ala350 is located in the Loop region, connecting the helix $\alpha 5$. Mutation of the alanine residue at this position to a serine may lead to the extensive formation of new hydrogen bonds, altering the flexibility of the loop and thus affecting catalytic activity [40]. Analysis showed that the thermal stability of A350S was reduced, with a half-life of 17.33 min at $60{ }^{\circ} \mathrm{C}$, less than two-fifths of that of WT DgoKerA (46.52 min). The high proportion of hydrophobic amino acids in the mature region of thermotolerant keratinase was found to be the molecular basis for its thermal stability [41,42]. The alanine residues are more hydrophobic than the serine residues, and therefore the reduced thermal stability of mutant A350S may be related to the hydrophobicity of residues in the mature region. As A350S is significantly more active against soluble keratinase than DgoKerA, subsequent modifications can be carried out on the basis of mutant A350S to improve its thermal stability.

\section{Conclusions}

In this study, the keratinase DgoKerA was identified from the genome of D. gobiensis I- 0 of Gobi origin. The amino acid sequence analysis revealed that DgoKerA was 58.68\% identical to the keratinase MtaKerA from M. thermophila WR-220 and 40.94\% identical to the classical BliKerA sequence from B. licheniformis PWD-1. In vitro enzyme activity analysis showed that DgoKerA exhibited an optimum temperature of $60^{\circ} \mathrm{C}$, an optimum $\mathrm{pH}$ of 7 and a specific enzyme activity of $51,147 \mathrm{U} / \mathrm{mg}$. The molecular modification of DgoKerA was also carried out using site-directed mutagenesis, in which the mutant A350S enzyme activity was increased by nearly $30 \%$, and the results provide a theoretical basis for the development and optimization of keratinase applications.

Author Contributions: Conceptualization, Y.M., Z.Z. and J.W.; methodology, Y.M., Y.T.; validation, Y.M. and X.Z.; formal analysis, Y.M. and X.Z.; investigation, Y.M. and Y.T.; resources, Z.Z. and J.W.; data curation, Y.M., Y.T. and X.Z.; writing—original draft preparation, Y.M.; writing-review and editing, Z.Z. and J.W.; visualization, Y.M.; supervision, Z.Z. and J.W.; project administration, Z.Z. and J.W.; funding acquisition, Z.Z. and J.W. All authors have read and agreed to the published version of the manuscript.

Funding: This work was supported by National Key R\&D Program of China (Nos. 2018YFA0901000, 2018YFA0901003), the National Transgenic Major Program of China (No. 2019ZX08010-004), We also appreciate the support of the Agricultural Science and Technology Innovation Program of CAAS (No. CAAS-ZDRW202009).

Institutional Review Board Statement: Not applicable.

Informed Consent Statement: Not applicable.

Data Availability Statement: Data sharing not applicable.

Acknowledgments: Special thanks Min Lin for constructive suggestions on the revision of the manuscript.

Conflicts of Interest: The authors declare no conflict of interest.

\section{References}

1. Lange, L.; Huang, Y.; Busk, P.K. Microbial decomposition of keratin in nature-a new hypothesis of industrial relevance. Appl. Microbiol. Biotechnol. 2016, 100, 2083-2096. [CrossRef]

2. Li, Q. Progress in Microbial Degradation of Feather Waste. Front. Microbiol. 2019, 10, 2717. [CrossRef] [PubMed] 
3. Nnolim, N.E.; Udenigwe, C.C.; Okoh, A.I.; Nwodo, U.U. Microbial Keratinase: Next Generation Green Catalyst and Prospective Applications. Front. Microbiol. 2020, 11, 580164. [CrossRef]

4. Gupta, R.; Ramnani, P. Microbial keratinases and their prospective applications: An overview. Appl. Microbiol. Biotechnol. 2006, 70, 21-33. [CrossRef] [PubMed]

5. Gupta, R.; Rajput, R.; Sharma, R.; Gupta, N. Biotechnological applications and prospective market of microbial keratinases. Appl. Microbiol. Biotechnol. 2013, 97, 9931-9940. [CrossRef]

6. Lin, X.; Kelemen, D.W.; Miller, E.S.; Shih, J.C. Nucleotide sequence and expression of kerA, the gene encoding a keratinolytic protease of Bacillus licheniformis PWD-1. Appl. Environ. Microbiol. 1995, 61, 1469-1474. [CrossRef]

7. Wu, W.L.; Chen, M.Y.; Tu, I.F.; Lin, Y.C.; EswarKumar, N.; Chen, M.Y.; Ho, M.C.; Wu, S.H. The discovery of novel heat-stable keratinases from Meiothermus taiwanensis WR-220 and other extremophiles. Sci. Rep. 2017, 7, 4658. [CrossRef]

8. Foophow, T.; Tanaka, S.; Koga, Y.; Takano, K.; Kanaya, S. Subtilisin-like serine protease from hyperthermophilic archaeon Thermococcus kodakaraensis with N- and C-terminal propeptides. Protein Eng. Des. Sel. 2010, 23, 347-355. [CrossRef]

9. Shinde, U.; Thomas, G. Insights from bacterial subtilases into the mechanisms of intramolecular chaperone-mediated activation of furin. Methods Mol. Biol. 2011, 768, 59-106. [CrossRef] [PubMed]

10. Fang, Z.; Zhang, J.; Liu, B.H.; Du, G.C.; Chen, J. Insight into the substrate specificity of keratinase KerSMD from Stenotrophomonas maltophilia by site-directed mutagenesis studies in the S1 pocket. RSC Adv. 2015, 5, 74953-74960. [CrossRef]

11. Wong, W.; Wijeyewickrema, L.C.; Kennan, R.M.; Reeve, S.B.; Steer, D.L.; Reboul, C.; Smith, A.I.; Pike, R.N.; Rood, J.I.; Whisstock, J.C.; et al. S1 pocket of a bacterially derived subtilisin-like protease underpins effective tissue destruction. J. Biol. Chem. 2011, 286, 42180-42187. [CrossRef]

12. Foophow, T.; Tanaka, S.; Angkawidjaja, C.; Koga, Y.; Takano, K.; Kanaya, S. Crystal structure of a subtilisin homologue, Tk-SP, from Thermococcus kodakaraensis: Requirement of a C-terminal beta-jelly roll domain for hyperstability. J. Mol. Biol. 2010, 400, 865-877. [CrossRef] [PubMed]

13. van den Burg, B. Extremophiles as a source for novel enzymes. Curr. Opin. Microbiol. 2003, 6, 213-218. [CrossRef]

14. Dalmaso, G.Z.L.; Lage, C.A.S.; Mazotto, A.M.; Dias, E.P.D.; Caldas, L.A.; Ferreira, D.; Vermelho, A.B. Extracellular peptidases from Deinococcus radiodurans. Extremophiles 2015, 19, 989-999. [CrossRef] [PubMed]

15. Zhang, Y.G.; Lu, W.; Wang, J.; Chen, M.; Zhang, W.; Lin, M.; Zhou, Z.F.; Liu, Z. Characterization of EstDR4, a Novel Cold-Adapted Insecticides-Metabolizing Esterase from Deinococcus radiodurans. Appl. Sci. 2021, 11, 1864. [CrossRef]

16. Kiran, T.; Asad, W.; Siddiqui, S.; Ajaz, M.; Rasool, S.A. Industrially important hydrolytic enzyme diversity explored in stove ash bacterial isolates. Pak. J. Pharm. Sci. 2015, 28, 2035-2040.

17. Khoshnevis, N.; Rezaei, S.; Forootanfar, H.; Faramarzi, M.A. Efficient Keratinolysis of Poultry Feather Waste by the Halotolerant Keratinase from Salicola Marasensis. Iran. J. Pharm. Res. 2019, 18, 1862-1870. [CrossRef]

18. Tang, Y.; Guo, L.Z.; Zhao, M.M.; Gui, Y.; Han, J.H.; Lu, W.; Dai, Q.L.; Jiang, S.J.; Lin, M.; Zhou, Z.F.; et al. A Novel Thermostable Keratinase from Deinococcus geothermalis with Potential Application in Feather Degradation. Appl. Sci. 2021, 11, 3136. [CrossRef]

19. Zhang, R.Y.; Huang, Y.; Qin, W.J.; Quan, Z.X. The complete genome of extracellular protease-producing Deinococcus sp. D7000 isolated from the hadal region of Mariana Trench Challenger Deep. Mar. Genom. 2021, 57, 100832. [CrossRef]

20. Zeng, Y.H.; Shen, F.T.; Tan, C.C.; Huang, C.C.; Young, C.C. The flexibility of UV-inducible mutation in Deinococcus ficus as evidenced by the existence of the imuB-dnaE2 gene cassette and generation of superior feather degrading bacteria. Microbiol. Res. 2011, 167, 40-47. [CrossRef]

21. Yuan, M.; Chen, M.; Zhang, W.; Lu, W.; Wang, J.; Yang, M.; Zhao, P.; Tang, R.; Li, X.; Hao, Y.; et al. Genome sequence and transcriptome analysis of the radioresistant bacterium Deinococcus gobiensis: Insights into the extreme environmental adaptations. PLoS ONE 2012, 7, e34458. [CrossRef]

22. Yang, L.; Wang, H.; Lv, Y.; Bai, Y.; Luo, H.; Shi, P.; Huang, H.; Yao, B. Construction of a Rapid Feather-Degrading Bacterium by Overexpression of a Highly Efficient Alkaline Keratinase in Its Parent Strain Bacillus amyloliquefaciens K11. J. Agric. Food Chem. 2016, 64, 78-84. [CrossRef] [PubMed]

23. Ren, Y.; Luo, H.; Huang, H.; Hakulinen, N.; Wang, Y.; Wang, Y.; Su, X.; Bai, Y.; Zhang, J.; Yao, B.; et al. Improving the catalytic performance of Proteinase $\mathrm{K}$ from Parengyodontium album for use in feather degradation. Int. J. Biol. Macromol. 2020, 154, 1586-1595. [CrossRef]

24. Niehaus, F.; Bertoldo, C.; Kahler, M.; Antranikian, G. Extremophiles as a source of novel enzymes for industrial application. Appl. Microbiol. Biot. 1999, 51, 711-729. [CrossRef] [PubMed]

25. Eder, J.; Fersht, A.R. Pro-sequence-assisted protein folding. Mol. Microbiol. 1995, 16, 609-614. [CrossRef]

26. Shinde, U.; Li, Y.; Chatterjee, S.; Inouye, M. Folding pathway mediated by an intramolecular chaperone. Proc. Natl. Acad. Sci. USA 1993, 90, 6924-6928. [CrossRef]

27. Fang, Z.; Zhang, J.; Liu, B.; Jiang, L.; Du, G.; Chen, J. Cloning, heterologous expression and characterization of two keratinases from Stenotrophomonas maltophilia BBE11-1. Process Biochem. 2014, 49, 647-654. [CrossRef]

28. Zhou, C.; Qin, H.; Chen, X.; Zhang, Y.; Xue, Y.; Ma, Y. A novel alkaline protease from alkaliphilic Idiomarina sp. C9-1 with potential application for eco-friendly enzymatic dehairing in the leather industry. Sci. Rep. 2018, 8, 16467. [CrossRef]

29. Fang, Z.; Zhang, J.; Du, G.; Chen, J. Rational protein engineering approaches to further improve the keratinolytic activity and thermostability of engineered keratinase KerSMD. Biochem. Eng. J. 2017, 127, 147-153. [CrossRef] 
30. Brandelli, A.; Daroit, D.J.; Riffel, A. Biochemical features of microbial keratinases and their production and applications. Appl. Microbiol. Biotechnol. 2010, 85, 1735-1750. [CrossRef]

31. Hassan, M.A.; Taha, T.H.; Hamad, G.M.; Hashem, M.; Alamri, S.; Mostafa, Y.S. Biochemical characterisation and application of keratinase from Bacillus thuringiensis MT1 to enable valorisation of hair wastes through biosynthesis of vitamin B-complex. Int. J. Biol. Macromol. 2020, 153, 561-572. [CrossRef]

32. Pereira, J.Q.; Ambrosini, A.; Passaglia, L.M.P.; Brandelli, A. A new cold-adapted serine peptidase from Antarctic Lysobacter sp. A03: Insights about enzyme activity at low temperatures. Int. J. Biol. Macromol. 2017, 103, 854-862. [CrossRef]

33. Bouacem, K.; Bouanane-Darenfed, A.; Zarai Jaouadi, N.; Joseph, M.; Hacene, H.; Ollivier, B.; Fardeau, M.L.; Bejar, S.; Jaouadi, B. Novel serine keratinase from Caldicoprobacter algeriensis exhibiting outstanding hide dehairing abilities. Int. J. Biol. Macromol. 2016, 86, 321-328. [CrossRef]

34. Dukunde, A.; Schneider, D.; Lu, M.; Brady, S.; Daniel, R. A novel, versatile family IV carboxylesterase exhibits high stability and activity in a broad pH spectrum. Biotechnol. Lett. 2017, 39, 577-587. [CrossRef] [PubMed]

35. Navone, L.; Speight, R. Understanding the dynamics of keratin weakening and hydrolysis by proteases. PLoS ONE 2018, 13, e0202608. [CrossRef]

36. Peng, Z.; Mao, X.; Zhang, J.; Du, G.; Chen, J. Biotransformation of keratin waste to amino acids and active peptides based on cell-free catalysis. Biotechnol. Biofuels 2020, 13, 61. [CrossRef]

37. Pourjavaheri, F.; Pour, S.O.; Jones, O.A.H.; Smooker, P.M.; Brkljaca, R.; Sherkat, F.; Blanch, E.W.; Gupta, A.; Shanks, R.A. Extraction of keratin from waste chicken feathers using sodium sulfide and L-cysteine. Process Biochem. 2019, 82, 205-214. [CrossRef]

38. Gong, J.S.; Ye, J.P.; Tao, L.Y.; Su, C.; Qin, J.; Zhang, Y.Y.; Li, H.; Li, H.; Xu, Z.H.; Shi, J.S. Efficient keratinase expression via promoter engineering strategies for degradation of feather wastes. Enzym. Microb. Technol. 2020, 137, 109550. [CrossRef] [PubMed]

39. Zhang, Y.; Ultsch, M.; Skelton, N.J.; Burdick, D.J.; Beresini, M.H.; Li, W.; Kong-Beltran, M.; Peterson, A.; Quinn, J.; Chiu, C.; et al. Discovery of a cryptic peptide-binding site on PCSK9 and design of antagonists. Nat. Struct. Mol. Biol. 2017, 24, 848-856. [CrossRef] [PubMed]

40. Kumar, S.; Tsai, C.J.; Nussinov, R. Factors enhancing protein thermostability. Protein Eng. 2000, 13, 179-191. [CrossRef]

41. Sittipol, D.; Rodpan, S.; Ajingi, Y.S.; Lohnoo, T.; Lerksuthirat, T.; Kumsang, Y.; Yingyong, W.; Khunrae, P.; Rattanarojpong, T.; Pattanapanyasat, K.; et al. Identification, overexpression, purification, and biochemical characterization of a novel hyperthermostable keratinase from Geoglobus acetivorans. 3 Biotech 2021, 11, 2. [CrossRef] [PubMed]

42. Jakob, F.; Martinez, R.; Mandawe, J.; Hellmuth, H.; Siegert, P.; Maurer, K.H.; Schwaneberg, U. Surface charge engineering of a Bacillus gibsonii subtilisin protease. Appl. Microbiol. Biotechnol. 2013, 97, 6793-6802. [CrossRef] [PubMed] 